\title{
Fürsorge für arme Wöchnerinnen in Hamburg um 1800: die Beschreibung eines «Entbindungs-Winkels»
}

Von Mary Lindemann

Eine Accouchier-Anstalt in Hamburg wurde erst 1796 errichtet $^{1}$. Vorher existierten aber andere Möglichkeiten für unvermögende, obdachlose oder gar unverheiratete Schwangere, «freie Entbindung» zu erhalten. Schon seit dem Ende des siebzehnten Jahrhunderts erlaubten die Prätoren - die städtischen Polizei-Magistrate - die Niederkunft solcher Frauen innerhalb des kommunalen Gefängnisses, des sogenannten Spinnhauses ${ }^{2}$. Dort entband die Rathswehemutter (Raths-Hebamme) diese «Geschwächte» und die Stadt-Kasse deckte die dabei entstehenden Unkosten wie z.B. die Hebammen-Gebühren, die Kosten für Medikamente, das Wartegeld und dergleichen. Ursprünglich diente das Spinnhaus als Entbindungsort nur für schwangere Gefangene. Im Laufe des achtzehnten Jahrhunderts erweiterte sich der Umfang dieses Systems: Das Spinnhaus wurde zum Zufluchtsort für bedürftige und wohnungslose Schwangere. Außerdem bezahlten die Gotteskasten gelegentlich die Hebammen-Gebühren für die Entbindung ihrer sogeannten «Haus-Armen».

Die Spinnhaus-Einrichtung (wie auch die der Gotteskasten) blieb aber provisorisch, obgleich viele Frauen in diesem Haus gebaren. Dabei entstanden allerdings einige Nachteile für die dort versorgten Frauen. Erstens galt das Spinnhaus als ein «unehrliches» und deshalb «entehrendes» Haus. Zweitens mußten die Wöchnerinnen oft längere Zeit (von sechs bis achtzehn Monaten) im Hause bleiben, um ihre «Schulden» abzuarbeiten. Beide Umstände erregten verständlicherweise Abscheu für das Haus und für das ganze System und beeinträchtigten dadurch erheblich dessen mildtätigen Nutzen ${ }^{3}$.

1788 wurde die Allgemeine Armenanstalt gegründet. Als Teil ihres weitläufigen Programmes bot sie seither den bei ihr eingetragenen Armen «freie Entbindung» an. (Außerdem bestand das Spinnhaussystem weiter.) Die Armen entbanden danach in ihren Wohnungen und erhielten die Hilfe von städtisch vereidigten und bei der Armenanstalt angestellten Hebammen ${ }^{4}$. Die Armenanstalt bezahlte die Gebühren der Hebammen (in der Regel 3 Mark) und, falls nötig, unterstützte die Frauen und ihre Familien weiter mit Kranken- und Speisegeldern ${ }^{5}$. Durch dieses System wurden 
verheiratete und ortsansässige Schwangere recht gut versorgt. Die Lage der unverheirateten, durchreisenden oder obdachlosen Mütter blieb dagegen weniger erfreulich.

1796 errichtete die Armenanstalt ihren eigenen Entbindungs-Saal in einem vom städtischen Zuchthause gemieteten Anbau. Dieses Institut nahm ausdrücklich nur obdachlose Schwangere und uneheliche Mütter auf; verheiratete Frauen genossen nach wie vor den Vorteil freier Entbindung zu Hause $^{6}$. Nach dem Wochenbett wurde von gesunden Wöchnerinnen erwartet, sogar verlangt, daß sie in einer durch die Armenanstalt verabredeten Stellung als Ammen dienten. Diese Entbindungsanstalt erfüllte weder die Aufgaben einer Schule für Hebammen, noch bot sie Unterricht in der Geburtshilfe für Studenten der Medizin oder der Wundarzneikunst an. Mit der Verwaltung dieses Saales wurde ein Sonderausschuß der MedizinalDeputation der Armenanstalt beauftragt. Normale Geburten wurden von einer in der Anstalt residierenden Hebamme überwacht; ein Arzt und ein Wundarzt halfen in schwierigen und gefährlichen Fällen. Die zwei ersten ärztlichen Direktoren waren die Geburtshelfer und Armenärzte Cornelius Jaenisch $^{7}$ (Direktor 1796-1810) und Georg Kerner (1810-1812). Dieser schrieb 1810 ein kleines, jedoch sehr lehrreiches Büchlein «Über das Hamburgische Entbindungshaus und das Entbindungswesen der Armenanstalt» (Hamburg 1810). ${ }^{8}$

Diese Entbindungs-Anstalt schloß aber Schwangere, die mit venerischen, krätzigen oder «andern verdächtigen Krankheiten» behaftet waren, völlig aus. Wegen des Platzmangels im Zuchthaus-Anbau und wegen der Kontagiosität ihres Leidens wurden diese Frauen entweder «in ein außerhalb des Hauses in der Nähe belegenes Zimmer, oder im Wandsbeckerhof in der Steinstraße ... zur Cur und Entbindung übergeben». ${ }^{9}$ Bald jedoch erwiesen sich die Zustände innerhalb dieser Nebenstellen als allzu erbärmlich und führten schließlich sogar zu einer Untersuchung. Eine Kommission der Armenanstalt, welche den Wandsbeckerhof besuchte, entdeckte hier die schlimmsten Unzulänglichkeiten. Am 8. Mai 1800 legte sie dem ArmenKollegium ihren ausführlichen - hier abgedruckten - Bericht vor. Dieser dient, wenn auch nur in bedingtem Maße, als eine glaubwürdige Beschreibung derartiger privater Einrichtungen. Diese war typisch für jene «privaten» Gebärhäuser, die sogenannten «Entbindungs-Winkel», welche vor der Gründung der Entbindungs-Anstalt den unehelichen Schwangeren oft den einzigen Zufluchtsort boten; allerdings nur dann, wenn diese Frauen über einige Geldmittel verfügten. Die Ärmeren waren vermutlich noch viel 
schlechter versorgt. Johann Jakob Rambach (Stadt-Physicus von 1804 bis 1812) beschrieb 1801, wie (vor der Gründung der Entbindungs-Anstalt)

... [die] Schwangeren, denen es an zulänglichen Mitteln zur Privatentbindung gefehlt, in eine elende schmützige Hütte verwiesen [waren], so sie den Händen eines wücherischen Gesindels übergeben, vergebens Hülfe und Pflege suchten und nicht einmal Mitleid fanden. Die dort geborenen Kinder aber sturben wahrscheinlich alle, da es einer sorgfältigsten Nachfrage nicht gelang, auch nur ein Kind auszuforschen, welches dort geboren war ${ }^{10}$.

Die Frage ist, ob es sich hier um eine Beschreibung des Wandsbeckerhofes handelte.

\section{Der Bericht an das Große Armenkollegium}

«Herr Vorsteher von Axen ${ }^{11}$ zeigte dem Armen-Collegio eine EntbindungsAnstalt an, welche in der Steinstraße in Wandsbecker Hoff unter der Aufsicht des Spinnhauses von der Familie Rusch verwaltet wurde, und worin diejenigen Personen welche Unreinlich mit Krätze ${ }^{12}$ oder andern verdächtigen Krankheiten behaftet wären und in der Entbindungs-Anstalt der Armen-Ordnung ${ }^{13}$ aus dieser Ursache nicht aufgenommen werden können durch Hülfe der Prätur und auf Kosten dieser Casse dort freyes Wochenbett erhielten.

Diese Anstalt deren Abscheulichkeit in Rücksicht des elenden Locales der medicinischen Hülfe und der administration die Herrn Senator Günther ${ }^{14}$ und ihm schon seit einiger Zeit bekannt gewesen sey - habe durch die Anzeige unsers thätigen Pflegers Herrn Nohr ${ }^{15}$ - durch die Aufmerksamkeit der Pfleger, in den dortigen Gegenden, und durch die Klagen der Herrn Prediger ${ }^{16}$ des Jacobi Kirchspiels die dort Sterbende beygestanden hätten eine größere publicitaet erhalten. Reverent in Gesellschaft der Herren Vorsteher Prösch ${ }^{17}$, Dr. Jaenisch und Herrn Nohr hätten sich von der Wahrheit der von Herrn Nohr an die medicinal Deputation ${ }^{18}$ darüber gemachten Berichts durch den Augenschein überzeugt, und glaubten das zur Ehre unserer Stadt eine solche Anstalt nicht länger daselbst geduldet werden müße.

Um dem Armen-Collegio mit diesen elenden Institute bekannter zu machen, wolle er eine data anführen, welche die Aufhebung deßelben verfertigen würden. 
Sie fänden daß die ganze Größe dieser dazu bestimmte Wohnung in einer zerfallnen beynahe fensterlosen Bude, - worin eine Stube von circa $15 \mathrm{Fuß}$ in quadrat, - ein kleiner Vorplatz mit Küche, und einen Boden von ohngefahr $25 \mathrm{Fu}$ in Quadrat bestünden. Die Treppe zum Boden auf dem bald nach der Entbindung die Wöchnerinnen geschafft wurden, sey so schmal und finster, daß kaum gesunde Personen hinaufsteigen könnten. - Der Boden könne natürlich nicht geheizt werden, und da er unter einen undichten Dache liege, so wären die Wöchnerinnen jeder Witterung ausgesetzt.

Gestanck, Schmutz - und ein hoher Grad der Unreinlichkeit wäre in dieser Hütte abscheulich.

Die eigentlichen Bewohner dieser Bude wären - Rusch und Frau 70 und $66 \mathrm{Jahr}$.

Die Ruschen welche die ganze Öconomie dieser Anstalt vorstehe, wäre ein zänkisches versoffenes Weib, die beständig die Zeichen der Prügeley mit den Nachbarn an sich trage.

Wachsmutt. 36 Jahre dessen Frau der Ruschen ihre Tochter 35 Jahre alt, währe beynahe epileptisch, 2 Kinder von letztern 5 und 3 Jahr. Ein altes gebrechliches Weib, welches in der Wirthschaft hülfe, und zuweilen noch den versoffne Sohn der Ruschen welcher auf einen Cattun Winckel arbeite und hier sehr oft die Nächte bliebe.

Für alle diese Bewohner sey das Locale kaum gros genug und doch wären die zufälligen Bewohner. Die Schwangern und Wöcherin die ihr von der Praetur zugesandt wurden und diejenigen welche sie oft dennoch für KostGeld aufnehme - 6 oder 8 Personen in den engen Zimmer werden die Schwangern unter den Geschrey der Kinder - den Lärmen und Gezänck der Weiber - den Zuckungen der epileptischen - und unter den Geächze der Kreisenden Entbunden.

Höchst wahrscheinlich starben alle diese Kinder bald nach der Geburt, den aller angewandten Mühe ohngeachtet habe Referent keinen in dieser Anstalt gebohren Kinder auffragen können ${ }^{19}$.

Die Entbindung geschehe von einer Lohn-Frau der Raths-Wehemutter ${ }^{20}$, und es sey nicht selten das dort Wöcherin wärend und nach der Geburt stürben. medicinische Hülfe erhielten sie fast keine andre als die sie von der Ruschen empfingen.

So schlecht dieses Institut verwaltet würde und so wenig Nutzen auch die Schwangern und Wöcherin von dieser anscheinenden Hülfe hätten, so wäre diese Art der Unterstützung doch sehr kostbar.

Den nach einer hiebey zu producirende Rechnung die Referent von den 
Herrn Senator Günther zu diesen Zweck in Abschrift erhalten hätte, habe der Herr Senator Günther aus den Prätur Casse für das Jahr 1799 - für 21. Entbindungen 646 [Mark] 15 [Schillinge] bezahlt.

Der Durchschnitt jeder Entbindung habe daher dem Staate 30 [Mark) 13 [Schillinge] gekostet, in der Entbindungs-Anstalt der Armen-Ordnung kostete jedes Wochenbett noch keine dreyzig [Mark] ${ }^{21}$ - Nach den Geständniß der Ruschen müßte jede Person in diesen Institute wöchentlich noch 1 [Mark] zu den Kostgeld aus eignen Mitteln zulegen.

So sehr es auch der Wunsch des Referenten schon lange gewesen wäre dem Armen-Collegio über die Aufhebung dieses Instituts Vorschläge zu machen, so haben doch der beschränkte Raum in unsrer Stadt und der daraus entstehende Mangel an einen schicklichen Locale welches er-alle angewandten Mühe ohngeachtet nicht habe ausfinden können - ihm davon abzuhalten. Itzt habe er einige Aussichten daß dieses Hinderniß abgeholfen werden könne.»

Wahrscheinlich als Folge dieses Berichts wurde das Entbindungs-Institut «durch einen, ihm vom Zuchthaus-Collegio zu diesem Behuf überlassenen, daran gränzenden Saal, um 6 Betten (4 für Schwangere und 2 für Wöchnerinnen) vergrößert ...» Trotzdem blieb der in dem Zuchthaus vorhandene Platz sehr beschränkt, und es war nicht möglich, ein völlig abgetrenntes, isoliertes Zimmer für venerische und krätzige Wöchnerinnen einzurichten. Für die Unterbringung dieser

ist ferner eine Frau aufgefunden, welche in der Neustädter Neustraße no. 57 wohnt und sich Schmuck nennt, welche ...2 Betten in einem geräumigen Locale stellen, diese Personen, auf Anweisung des Vorstehers der Entbindungs-Anstalt, gegen ein wöchentliches Kostgeld von 3 Mark, während ihrer Schwangerschaft und Wochenbette, pflegen und beköstigen wird; $\ldots{ }^{22}$

In der «Nachricht» der Allgemeinen Armenanstalt gab man zu, daß diese getroffenen Maßnahmen keineswegs vollkommen waren; versicherte jedoch zugleich, sie könne

aber nie ausarten, weil sie unter der Aufsicht des Vorstehers der Entbindungs-Anstalt steht, von dem die Empfehlung und die Bezahlung erfolgt und in medizinischer Rücksicht, unter der Fürsorge der Bezirks-Ärzte ist, und die Entbindung selbst durch die Bezirks-Wehemutter besorgt wird. ${ }^{23}$ 
Nach diesen dringend benötigten Änderungen und Verbesserungen, meinte man, es sei

jetzt hinlänglich für alle unverheiratete, schwangere Personen in Hamburg gesorgt, und das Unwesen mit der traurigen Entbindungs-Anstalt im Wandbecker Hof, wird ganz aufhören können. ${ }^{24}$

1821, als das Zuchthaus so baufällig geworden war, daß das Gebäude abgebrochen werden mußte, wurde die Anstalt im Kurhaus ${ }^{25}$ untergebracht.

Erst 1857 bekam dieses Institut ein eigenes Haus und wurde danach dem Armen-Kollegium, dem Gesundheitsrat und der Polizei zu gemeinsamer Verwaltung übergeben.

\section{Anmerkungen}

1 Einen kurzen Überblick über die Geschichte der Geburtshilfe und des Entbindungswesens in Hamburg bietet H.Dietel: «Lucina Hamburgensis. Studie zur Geschichte der Geburtshilfe in Hamburg», Hamburg 1964. Die Geschichte des hamburgischen Hebammenwesens ist neuerdings in einer medizin-historischen Dissertation von Christoph Axmann behandelt worden.

2 Siehe Adolf Streng: «Geschichte der Gefängnisverwaltung in Hamburg von 1622-1872», Hamburg 1890, S. 71-101; vgl. Andreas Ehrenfried Martens: «Das hamburgische CriminalGefängnis genannt: Das Spinnhaus und die übrigen Gefängnisse der Stadt Hamburg nach ihrer innern Beschaffenheit und Einrichtung beschrieben, nebst einigen Ansichten und Ideen über Verbesserung ähnlicher Anstalten», Hamburg 1823.

${ }^{3}$ Der «Extractus Protocolli Senatus Hamb. wegen der ins Spinnhaus zu Haltung der Wochen geschickten Weibs-Personen», 1770 spezificirte, daß

künftighin diejenigen liederliche Weibsbilder, welche von Ihnen [den Prätoren], zu Haltung der Wochen, nach dem Spinnhaus geschickt werden, nach deren Entbindung nicht sofort aus dem Spinnhause zu dimitteren, sondern die Verfügung zu machen, daß selbige, nach Befinden und der Größe ihres Verbrechens, annoch etwa ein halb Jahr im Spinnhause bleiben, und daselbst zur Arbeit angehalten werden.

In Johann Friedrich Blank (Hrsg.): «Sammlung der von E. Hochedlen Rathe der Stadt Hamburg so wol zur Handhabung der Gesetze und Verfassungen als bey besonderen Eräugnissen in Bürger- und Kirchlichen, auch Cammer-, Handlungs-, und übrigen PoliceyAngelegenheit und Geschäften vom Anfange des siebzehnten Jahr-Hunderts bis auf die itzige Zeit ausgegangenen allgemeinen Mandate, bestimmten Befehle und Bescheide, auch beliebten Aufträge und verkündigten Anordnungen», Bd.6, Hamburg 1774, S. 325.

4 Siehe insbesondere $\S 58$ «Von der Fürsorge der armen Wöchnerinnen» und $\S 61$ «Von der Fürsorge für nicht eingezeichnete Wöchnerinnen» der «Einleitung zur Administration der hamb. Armenanstalt in besonderer Rücksicht auf die Geschäfte der Herrn Bezirks-Vorste- 
her», Hamburg o.d., und «Instruction für die bei der hamburgischen Armen-Anstalt anzustellenden Wehemütter», Hamburg o.d. (ca. 1790-91), beide in Staatsarchiv Hamburg, Allgemeine Armenanstalt I (StAHbg, AAI), Nr.100. Instruktion betr. die Armenkrankenpflege, insbesondere Ärzte, Wundärzte. Apotheker, Speisewirte, Wehemütter, Krankenwärterinnen, 1788-1796.

${ }^{5}$ «Bericht von 1st Jan.-Ende Dez. 1796», StAHbg, AAI, Nr. 117. Jahresbericht und Material betr. die Verwaltung der Entbindungsanstalt. Siehe auch: «Nachricht an Hamburgs wohlthätige Einwohner über den Fortgang der Armenanstalt», Nr.19, April 1796, S. 44.

${ }^{6}$ Ebenda.

${ }^{7}$ Dr. Med. Cornelius Jaenisch (1753-1810) war ein vornehmer hamburgischer Geburtshelfer. Er hat eine sehr ausgedehnte Praxis und fungierte auch als Arzt der Allgemeinen Armenanstalt. Sein Vater (1707-1781) und sein Bruder (1751-1830) hießen beide Gottfried Jakob und waren ebenfalls Ärzte. Cornelius Jaenisch und sein Bruder dienten als ArmenÄrzte der «Medizinischen Anstalt für kranke Haus-Armen in Hamburg», die von 1779 bis 1788 arbeitete. August Hirsch (Hrsg.): «Biographisches Lexikon der hervorragender Ärzte aller Zeiten und Völker vor 1880», 5 Bde., 3 Auflage, Berlin 1929-1934, und Hans Schröder et al. (Hrsg.): «Lexikon der hamburgischen Schriftsteller bis zur Gegenwart», 8 Bde., Hamburg 1851-1883.

8 Jetzt nachgedruckt in Hedwig Voegt (Hrsg.):

«Georg Kerner, Jakobiner und Armenarzt. Reisebriefe, Berichte, Lebenszeugnisse», Berlin [Ost] 1978, S. 371-384. Kerner (1770-1812), der 1791 auf der Karlsschule die medizinische Doktorwürde erlangen hatte, spielte während der Französischen Revolution eine Rolle in Paris und ging 1803 nach Hamburg. 1812 wurde er Arzt am Zuchthause ernannt, starb aber kurz danach an Typhus (Schröder: «Schriftsteller Lexikon»).

${ }^{9}$ StAHbg, AAI, Nr.9 Protokoll des großen Armenkollegiums, 10. März 1796.

${ }^{10}$ Johann Jakob Rambach: «Versuch einer physisch-medizinischen Beschreibung von Hamburg», Hamburg 1801, S.426-427.

11 Otto von Axen (1757-1831) wurde 1791 Armenvorsteher. Ab 1796 war er das mit der Verwaltung und der täglichen Administration der Entbindungsanstalt beauftragte Mitglied des großen Armen-Kollegiums (Schröder: «Schriftsteller Lexikon»).

12 Krätze = Skabies. Diese Hautkrankheit wütete in den unteren Schichten der Bevölkerung und war besonders tief eingewurzelt, sogar endemisch und fast unausrottbar in dem städtischen Zuchthaus und im Waisenhause.

${ }^{13}$ Die «Neue Armen-Ordnung der Stadt Hamburg» rief die neue Allgemeine Armenanstalt ins Leben (in Christian Daniel Anderson: «Sammlung Hamburgischer Verordnungen», Band 2, Hamburg 1789, S.342-354). Mitglieder der Hamburgischen Patriotischen Gesellschaft vor allem Kaspar von Voght, Johann Arnold Günther und Johann Georg Büsch - waren weitgehend verantwortlich für ihre Entstehung und die Gestaltung ihres Programmes. Eine wichtige Aufgabe dieser Anstalt war die medizinische Versorgung der ärmeren Bevölkerung, welche durch die Medizinal-Deputation der Anstalt gewahrleistet wurde. Vgl. Lotte Koch: «Wandlungen der Wohlfahrtspflege im Zeitalter der Aufklärung», Erlangen 1933, S. 155-159, und Caspar von Voght: «Gesammeltes aus der Geschichte der Hamburgischen Armen-Anstalt während ihrer fünfzigjährigen Dauer», Hamburg 1838.

${ }^{14}$ Johann Arbold Günther (1755-1805) studierte Rechtswissenschaft in Göttingen und wurde 1778 Lizentiat der Rechte. Seit 1781 war er Erster Sekretar der Patriotischen Gesellschaft. 
Er war auch Mitbegründer der Allgemeinen Armenanstalt und einer ihrer ersten Vorsteher. 1792 wurde er zum Rath gewählt und als Ratsherr fungierte er als «Armen-Herr» des dritten Armen-Bezirks. Jahrelang leitete dieser eifrige Mann die Medizinal-Deputation und wirkte als Mitglied derselben bis zu seinem Tode mit. 1795 entwarf er fast eigenhändig geeignete Vorschläge für eine gänzliche Revision der Medizinalgesetze in Hamburg, die aber erst 1818 fruchtete. Siehe Schröder: «Schriftsteller Lexikon»; Hermann Gustav Gernet: «Mitteilungen aus der älteren Medicinalgeschichte Hamburgs. Kulturhistorische Skizee auf urkundlichem und geschichtlichem Grunde», Hamburg 1869, S.333-334, und Heinrich Rodegra: «Das Gesundheitswesen der Stadt Hamburg im 19. Jahrhundert unter Berücksichtigung der Medizinalgesetzgebung (1586-1818-1900)», Wiesbaden 1979, S.44-48.

15 Johann Anton Nohr wohnte in der Breitenstraße Nr.64. 1800 diente Nohr bereits zum drittenmal - d.h. neun Jahre - als Armenpfleger im dritten Armenbezirk, obwohl er im zweiten Bezirk wohnte («Hamburgischer Staats-Kalender auf das 1800ste Jahr Christi ..., darinn der gegenwärtige Staat der Stadt Hamburg», Hamburg 1800),

${ }^{16}$ Im Jahre 1800 war Christian Ludewig Gerling Haupt-Pastor zu St. Jakobi. Seit 1784 war er Seniore des Hamburgischen Reverend Ministeriums. Drei untergeordneten Predigter standen ihm bei: der Archi-Diaconus Nicolaus Joachim Guil. Evers, der zweite Diaconus Bernhard Klefeker, und der dritte Diaconus Hermann Rentzel. Wahrscheinlich handelte es sich hier um einer dieser Untergordneten («Staatskalender 1800»).

${ }^{17}$ Luer Anton Prösch (1758-1826) war Armen- und Schulvorsteher in dem 3. Armen-Bezirk seit 1792. Er wurde zum Kämmerabgeordneten 1800 und zum Rathsherrn 1810 gewählt. Die Steinstraße lag innerhalb seiner Bezirks-Hälfte. Er gehörte auch der MedizinalDeputation der Allgemeinen Armenanstalt an.

${ }^{18}$ Die Medizinal-Deputation trug die Verantwortlichkeit für die medizinische Versorgung der Armen. Die anderen Mitglieder dieser Deputation im Jahre 1800 waren von Axen (als verwaltender Vorsteher), Voght, Günther und der Oberalt Johann Friedrich Tönnies («Staatskalender 1800»).

${ }^{19}$ Vgl. Rambach: «Versuch einer Beschreibung», S.426-427.

${ }^{20}$ Die Raths-Wehemutter (oder Raths-Hebamme) war durch den Rath beeidigt und angestellt. U.a. sorgte sie für die kostenlose Entbindung der Obdachlosen, der Insassen der Armenhäuser und der Gefangenen. Auch diente sie als «Ober-Hebamme», welche die Überwachung aller andern Hebammen in der Stadt übernahm. Die hier erwähnte LohnFrau war wahrscheinlich einer ihrer Lehrlinge (siehe Johann Klefeker: «Sammlung der Hamburgischen Gesetze und Verfassungen in Bürger- und Kirchlichen, auch Cammer-, Handlungs-, und übrigen Policey-Angelegenheiten und Geschäften samt historischen Einleitungen», Band 12, Hamburg 1763, S.458-466.

${ }^{21} 1800$ kostete die Entbindung und Versorgung einer Wöchnerinn innerhalb der Entbindungs-Anstalt 25 Mark 5 Sch. 5 Pfg. («Nachricht» Nr. 28 [Januar 1801], S.279).

${ }^{22}$ Ebenda.

${ }^{23}$ Ebenda.

24 Ebenda.

${ }^{25}$ Vgl. Andreas Ehrenfried Martens: «Das Hamburgische Kurhaus und dessen Einrichtung», Hamburg 1822. Von besonderem Interesse ist hier der Anhang: «Die Entbindungs-Anstalt im Kurhause. Hamburg. ultimo December 1821.»

${ }^{26}$ Dietel: «Lucina Hamburgensis», S.21-26. 


\section{Summary}

In the eighteenth century, the establishment of lying-in wards and maternity hospitals significantly improved the quality of maternal care for poor and unwed mothers. This report, taken from the records of the Hamburg General Poor Relief, however, illustrates the often inhumane and even exploitive conditions which, before such institutions existed, had awaited poor and unwed mothers in the many privately-run lying-in hospitals.

Mary Lindemann, Dr.phil.

Herzog-August-Bibliothek

Postfach 1227

D-3340 Wolfenbüttel 